\title{
Prevalence and impact of depression and pain in neurology outpatients
}

\author{
L S Williams, W J Jones, J Shen, R L Robinson, M Weinberger, K Kroenke
}

J Neurol Neurosurg Psychiatry 2003;74:1587-1589

Background: We examined the prevalence and health related quality of life (HRQoL) of depression and/or pain in neurology outpatients.

Methods: Patients at outpatient clinics completed depression, pain, and HRQoL scales. Group comparisons between those with pain alone, depression alone, both conditions, and neither condition were done.

Results: Overall, pain was present in $2 / 3$ and depression in $1 / 3$ of patients. Pain with depression was present in $25 \%$; $75 \%$ of depressed patients had pain. These conditions had significant negative impact on mental and physical health status scores. The odds ratio (OR) for having pain was significantly increased in women (OR 2.0), those with depression (OR 2.4), and those with neuropathy/neuromuscular (OR 3.8) or pain syndromes (OR 4.8). The odds of having depression were increased in those with pain (OR 2.4) and with cognitive (OR 4.8) or cerebrovascular (OR 3.3) diagnoses. Neurologists were more likely to recognise and treat pain than depression.

Conclusions: Depression and pain are common in newly referred neurology outpatients and have substantial negative effects on patients' physical and mental health. Pain is more likely than depression to be recognised and treated by neurologists.

$\mathrm{P}$ ior studies have documented the high prevalence of depression and pain diagnoses in primary care clinics, but corresponding data for neurology outpatients are scarce. ${ }^{1-4}$ Patients referred to neurology clinics may be at high risk of depression and pain because of the chronic nature of many neurological conditions, pain associated with neuromuscular disease and headache, and simply by being selected for referral for their condition. Depressive and painful symptoms may often present simultaneously as depression associated pain or as comorbid conditions and, if so, are less likely to respond to treatment. ${ }^{56}$ Further, how often neurologists recognise and treat depression and pain in their outpatients is not well described. The aims of this study were to: (a) estimate the prevalence of depression and pain in patients newly referred to three neurology outpatient clinics; (b) assess the impact of these conditions on patients' health status; (c) identify patient characteristics associated with the presence of these conditions, and (d) assess the frequency of recognition and treatment of depression and pain symptoms.

\section{METHODS}

We identified consecutive newly referred patients to three hospital based general neurology outpatient clinics from January 2001 to August 2001. Patients with no cognitive or language barriers to communication were eligible. Patients referred to specialty clinics (for example epilepsy, Alzheimer's and multiple sclerosis) were excluded. Research assistants approached patients in the clinic, explained the study, and obtained informed consent. This study was approved by the local institutional review board.

Study patients completed a series of questionnaires in the clinic and at 3 and 12 month follow up; only baseline data are included in this report. Pain was measured with the Brief Pain Inventory (BPI). ${ }^{7}$ We used the Patient Health Questionnaire nine item depression module (PHQ-9) to assess depression symptoms. ${ }^{8}{ }^{9}$ Scores $\geqslant 10$ on the PHQ-9 represent clinically significant depression. ${ }^{9}$ Neurologists were blinded to the PHQ-9 and BPI scores unless the patient endorsed thoughts of death as a depression symptom. We used the Medical Outcomes Study Short Form 12 (MOS SF12) to measure health status. ${ }^{10}$ This scale provides composite measures of physical component summary (PCS) and mental component summary (MCS) health status. Normative United States population scores have been developed so that the median PCS and MCS score is 50 and lower scores indicate worse health status.

After the clinic visit, standardised clinic encounter forms and physicians' dictated notes were reviewed to determine the primary neurological diagnosis and physician recognition and treatment of pain and mental health diagnoses. Research assistants used an intentionally liberal standardised list of words, phrases, and treatments (for example continuing a treatment or referring for treatment by another provider) to assess physician recognition and treatment of depression and pain.

Patients were categorised into four groups: depression alone, pain alone, pain with depression, and neither. One way ANOVAs were conducted to test for mean differences of the SF-PCS and SF-MCS by depression and pain groups using the Tukey-Kramer method to adjust for pair wise comparisons. We also modelled depression and pain separately using logistic regression, with sex, age, clinic site, and diagnoses as covariates. $\chi^{2}$ Tests and odds ratios (ORs) were used to test the significance of independent variables. Possible interactions between independent variables were also evaluated in both models.

\section{RESULTS}

Of the 587 patients newly referred during the six months of enrollment, $483(82 \%)$ were enrolled in the study, $92(16 \%)$ refused participation, and $12(2 \%)$ were ineligible. Sample characteristics are shown in table 1.

The most common primary diagnoses at the close of the baseline visit (fig 1) were neuropathy or neuromuscular

Abbreviations: $B P I$, Brief Pain Inventory; $\mathrm{HRQ}$ L, health related quality of life; MCS, mental component summary; MOS SF-12, Medical Outcomes Study Short Form 12; PCS, physical component summary; PHQ-9, Patient Health Questionnaire, nine item 
Table 1 Patient demographic and health status scores

\begin{tabular}{|c|c|c|c|c|c|}
\hline & $\begin{array}{l}\text { Depression } \\
\text { alone }(n=39)\end{array}$ & $\begin{array}{l}\text { Pain alone } \\
(n=198)\end{array}$ & $\begin{array}{l}\text { Pain with depression } \\
\text { ( } n=122)\end{array}$ & Neither ( $n=124)$ & p Value* \\
\hline Age & 51.1 (SD 21) & 48.9 (SD 16) & 48.2 (SD 15.3) & 52 (SD 18) & 0.28 \\
\hline Percentage female & 38 & 56 & 61 & 43 & 0.008 \\
\hline Percentage Caucasian & 90 & 88 & 84 & 88 & 0.68 \\
\hline PCS score† & 42.5 (SD 10.5) & 36.1 (SD 10.7) & 31.3 (SD 8.9) & 46.8 (SD 10.1) & $<0.001$ \\
\hline MCS score & 37.8 (SD 10.6) & 50.3 (SD 9.5) & 34.2 (SD 12.3) & 51.6 (SD 8.9) & $<0.001$ \\
\hline Pain intensity & - & 4.5 (SD 2.0) & 6.1 (SD 2.1) & - & $<0.001$ \\
\hline Pain interference & - & 4.5 (SD 2.3) & $7.1(2.1)$ & - & $<0.001$ \\
\hline PHQ-9 & 13.2 (SD 4.1) & 4.5 (SD 2.9) & 16.0 (SD 5.0) & 3.3 (SD 2.9) & $<0.001$ \\
\hline \multicolumn{6}{|c|}{$\begin{array}{l}\text { "p Values are for overall tests ( } \chi^{2} \text { tests are used for percentage female and percentage white). } \\
\text { †All pair wise comparisons significant at } p<0.003 \text { except for "depression alone" group versus "neither" group. } \\
\text { †All pair wise comparisons significant at } p<0.001 \text { except for "pain with depression" group versus "depression } \\
\text { alone" group and "pain alone" group versus "neither" group. } \\
\text { Pair wise comparisons adjusted by Tukey-Kramer method. } \\
\text { MCS, mental component summary; PCS, physical component summary; PHQ-9, Patient Health Questionnaire, nine } \\
\text { item. }\end{array}$} \\
\hline
\end{tabular}

disease $(n=109)$ and headache $(n=101)$. The symptomatic diagnosis category $(n=61)$ included diagnoses codes such as dizziness and numbness. It is striking that pain (alone or with depression) is reported in at least $40 \%$ of all subjects regardless of diagnosis group.

The table also summarises baseline health status scores for patients with depression, pain, both depression and pain, or neither. Nearly two thirds of patients reported pain, one third screened positive for depression, and one fourth had both depression and pain. Of those with pain, 33\% were also depressed. Of those with depression, $75 \%$ also had pain. Using the PHQ-9 diagnostic algorithm, 50\% of those with depression ( $17 \%$ of the total sample) had major depression and $22 \%$ ( $13 \%$ of the total sample) had "other depression". The depression alone group was almost twice as likely to consist of men (62\% men $v 38 \%$ women), while the pain with depression group was almost twice as likely to consist of women $(61 \%$ women $v 39 \%$ men, $\mathrm{p}<0.01)$.

Patient health status was significantly affected by pain and depression. SF-12 PCS and MCS scores approached the norm national median score of 50 only in those without pain or depression. PHQ-9 scores were also higher in those with comorbid pain and depression than in those with depression alone. Further, pain intensity and pain interference scores were significantly higher in the group with pain and depression than in those with pain alone.

In logistic regression models, the odds of having pain were significantly increased in women (OR 2.0, 95\% CI 1.2 to 3.2 ), in those with depression (OR 2.4, 95\% CI 1.4 to 4.0 ), and in

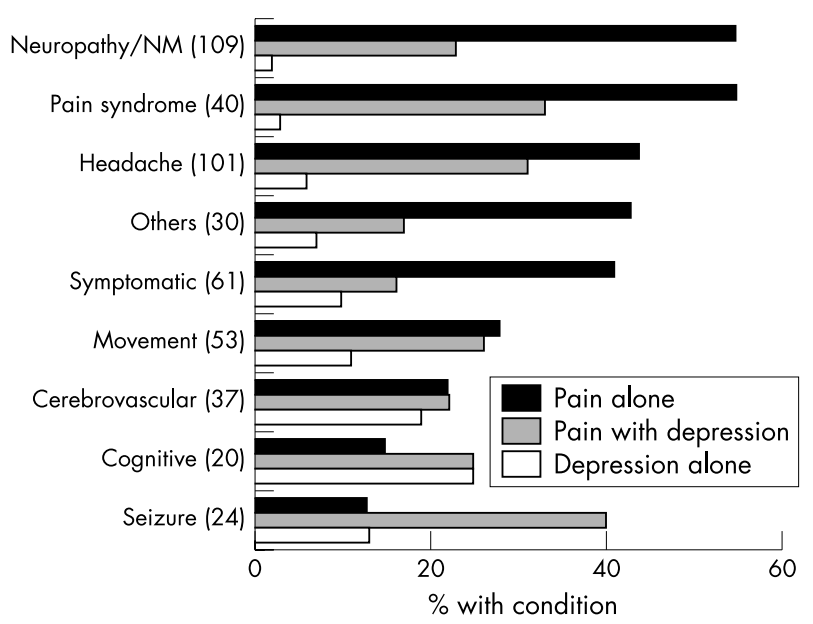

Figure 1 Depression and pain screening by primary diagnosis. those with neuropathy/neuromuscular (OR 3.8, 95\% CI 1.5 to 9.7) or pain syndrome (OR 4.8, 95\% CI 1.4 to 16.5 ) diagnoses. The odds of having depression were significantly increased in those with pain (OR 2.4, 95\% CI 1.5 to 3.9) and in those with cognitive (OR $4.8,95 \%$ CI 1.3 to 18.0 ) or cerebrovascular (OR $3.3,95 \%$ CI 1.0 to 10.6$)$ diagnoses.

Neurologists were more likely to recognise and treat pain than depression, but the presence of pain and depression together did not significantly affect recognition and treatment rates. Pain was recognised in $77 \%$ with pain and depression and $72 \%$ of those with pain alone $(p=N S)$, while depression was recognised in $48 \%$ of the pain with depression group and in $40 \%$ of those with depression alone $(p=N S)$. Similarly, patients with depression and pain were no more likely than those with either condition alone to receive a treatment plan. Overall, only $41 \%$ of patients screening positive for depression and 50\% with major depression received psychological treatment.

\section{DISCUSSION}

There is a high prevalence of depression and pain in patients referred to neurology outpatient clinics, with significant pain reported by more than two thirds of patients and depression present in one third. Although the prevalence varies somewhat by neurological diagnosis, no specific category of neurological disease is especially free of these conditions. Importantly, pain with depression is particularly common, being present in one in four newly referred outpatients in our sample. It is also clear that these conditions have a substantial adverse impact on patients' physical and mental health, with mean health status scores below the national median in all groups except those with neither pain nor depression. Despite differences in the process of neurology referral in the United States and the United Kingdom, these results are similar to a recent British study conducted by Carson and colleagues. ${ }^{1}$

Our study also demonstrates the strong relationship between depression and pain. This highlights the importance of recognising both conditions, because patients' health status and the perception of pain are significantly worse when both depression and pain are present. Moreover, this comorbidity has important implications for patient management, because other studies have documented greater difficulty in treating pain when depression is coexistant. ${ }^{5}$ The cross sectional nature of these data limits the ability to disentangle this complex relationship, but follow up data in this cohort are being accrued that may help to address this important issue.

Neurologists were more likely to recognise and treat pain than depression, but the presence of pain with depression did 
not significantly increase recognition and treatment. This is not surprising because it is well established that most patients with depression in primary care present with somatic rather than emotional complaints, ${ }^{11}{ }^{12}$ and because prior studies suggest that depression is less likely to be recognised and treated when physical comorbidities compete with depression symptoms during an outpatient visit. ${ }^{13}$ Because neurologists were not directly asked after each visit about their impression of either pain or depression, their assessment of these conditions may have been underestimated. Alternatively, because an intentionally liberal review of the written documentation of depression was employed, the recognition of depression may have been overestimated.

This study also documents a higher screening prevalence of depression in neurology clinics compared with primary care, where approximately $13-17 \%$ of patients screen positive for depression. ${ }^{8}{ }^{14-16}$ This higher prevalence has also been demonstrated in other specialty settings, including gastroenterology ${ }^{17}$ and rheumatology, ${ }^{18}$ and may indicate the need for increased training of specialists to recognise depression and for implementation of standardised screening programmes in settings where depression prevalence is high. Although we attempted to reduce the potential for selection bias by avoiding recruitment in identified specialty clinics, this may have made this cohort less generalisable to the private practitioner and reduced the assessment of common important conditions like epilepsy, a condition in which depression has been reported to be prevalent. ${ }^{19}$

Even if one argues that a neurologist (or any specialty consultant) is not the appropriate healthcare provider to treat depression in a given patient, recognising depression is important because it may affect treatment the neurologist prescribes. Further, neurologist recognition of depression may assist patients and their primary healthcare providers in obtaining optimal treatment for this debilitating disorder. Thus, neurologists might consider implementing simple, valid screening tests for depression in their newly referred patients, especially those with significant pain. ${ }^{20}$ Future studies should examine the longer term impact of these conditions on patient health status and satisfaction, the impact of depression on treatment for pain and other neurological symptoms, and the association between physician recognition and treatment of pain and depression and subsequent patient outcomes.

\section{ACKNOWLEDGEMENTS}

This work was supported by a contract from Eli Lilly and Company and was performed, in part, at the Regenstrief Institute for Health Care.

LW is supported by a Veterans Administration Research Career Development Award, Health Services Research.

\section{Authors' affiliations}

L S Williams, W J Jones, Departments of Neurology, Indiana University, Indianapolis, USA
J Shen, K Kroenke, Indiana University School of Medicine, Indianapolis, USA

L S Williams, K Kroenke, Regenstrief Institute for Health Care, Indianapolis, USA

R L Robinson, Eli Lilly and Company

M Weinberger, University of North Carolina, North Carolina, USA

Declaration of interest: KK has received research support and honoraria from Eli Lilli, Wyeth, and Pfizer.

Correspondence to: Dr S L Williams, Roudebush VAMC HSR\&D, 1481

West 10th Street, Indianapolis, IN 46202, USA;

Iwilliams@hsrd.va.iupui.edu

\section{REFERENCES}

1 Carson AJ, Ringbauer G, MacKenzie L, et al. Neurological disease, emotional disorder and disability: they are related. A study of 300 consecutive new referrals to a neurology outpatient department. J Neurol Neurosurg Psychiatry 2000;68:202-6.

2 Ekstrand J, O'Malley P, Labutta R, et al. Mental disorders in a neurology clinic setting [Abstract]. J Gen Intern Med 2000; 15(Suppl): 112.

3 Kirk C, Saunders M. Primary psychiatric illness in a neurological out-patient department in north east England. Acta Psychiatr Scand 1977;56:294-302.

4 Berlin RM, Ronthal M, Bixler EO, et al. Psychiatric symptomatology in an outpatient neurology clinic. J Clin Psychiatry 1983;44:204-6.

5 Fishbain DA, Cutler BR, Rosomoff HL, et al. Comorbidity between psychiatric disorders and chronic pain. Current Review of Pain 1998;2:1-10.

6 Fishbain DA, Cutler R, Rosomoff HL, et al. Chronic pain-associated depression: antecedent or consequence of chronic pain? A review. Clin J Pain 1997; 13:116-37.

7 Cleeland CS. Pain assessment in cancer. In: Osoba D, ed. Effect of cancer on quality of life. Boca Raton, Florida: CRC press, 1991:293-305.

8 Spitzer RL, Kroenke K, Williams JB. Validation and utility of a self-report version of PRIME-MD: the PHQ primary care study. Primary Care Evaluation of Mental Disorders, Patient Health Questionnaire. JAMA 1999;282: 17137-44.

9 Kroenke K, Spitzer RL, Williams JBW. The PHQ-9: validity of a brief depression severity measure. J Gen Intern Med 2001;16:606-13.

10 Ware JE, Kosinski M, Keller SD. A 12-item short-form health survey: construction of scales and preliminary test of reliability and validity. Med Care 1996;24:220-33.

11 Rost K, Nutting P, Smith J, et al. The role of competing demands in the treatment provided primary care patients with major depression. Archives of Family Medicine 2000;2:150-4.

12 Kroenke K. Studying symptoms: sampling and measurement issues. Ann Intern Med 2001;134:844-53.

13 Katon W, Sullivan M, Walker E. Medical symptoms without identified pathology: relationship to psychiatric disorders, childhood and adult trauma, and personality traits. Ann Intern Med 2001;134:917-25.

14 Williams JW Jr, Mulrow CD, Kroenke K, et al. Case-finding for depression in primary care: a randomized trial. Am J Med 1999;106:36-43

15 Whooley MA, Stone B, Soghikian K. Randomized trial of case-finding for depression in elderly primary care patients. J Gen Intern Med 2000;15:293-300.

16 Robbins JM, Kirmayer $\amalg$, Cathebras P, et al. Physician characteristics and the recognition of depression and anxiety in primary care. Med Care 1994;32:795-812.

17 O'Malley PG, Wong PWK, Kroenke K, et al. The value of screening for psychiatric disorders prior to upper endoscopy. Journal of Psychosomatic Research 1998:44:279-87.

18 O'Malley PG, Jackson JL, Kroenke K, et al. The value of screening for psychiatric disorders in rheumatology referrals. Arch Intern Med 1998; 158:2357-62.

19 Baker GA, Jacoby A, Buck D, et al. The quality of life of older people with epilepsy: findings from a UK community study. Seizure 2001;10:92-9.

20 Kroenke K, Taylor-Vaisey A, Dietrich AJ, et al. Interventions to improve provider recognition and management of mental disorders in primary care: a critical review of the literature. Psychosomatics 2000;41:39-52. 